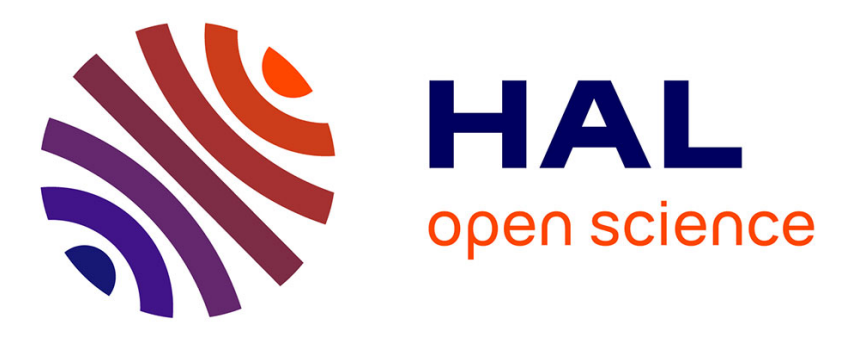

\title{
New predictive scheme for the control of LTI systems with input delay and unknown disturbances
}

Vincent Léchappé, Emmanuel Moulay, Franck Plestan, Alain Glumineau, Abdelhamid Chriette

\section{- To cite this version:}

Vincent Léchappé, Emmanuel Moulay, Franck Plestan, Alain Glumineau, Abdelhamid Chriette. New predictive scheme for the control of LTI systems with input delay and unknown disturbances. Automatica, 2015, 52, pp.179-184. 10.1016/j.automatica.2014.11.003 . hal-01093925

\section{HAL Id: hal-01093925 \\ https://hal.science/hal-01093925}

Submitted on 26 Jan 2016

HAL is a multi-disciplinary open access archive for the deposit and dissemination of scientific research documents, whether they are published or not. The documents may come from teaching and research institutions in France or abroad, or from public or private research centers.
L'archive ouverte pluridisciplinaire HAL, est destinée au dépôt et à la diffusion de documents scientifiques de niveau recherche, publiés ou non, émanant des établissements d'enseignement et de recherche français ou étrangers, des laboratoires publics ou privés. 


\title{
New predictive scheme for the control of LTI systems with input delay and unknown disturbances
}

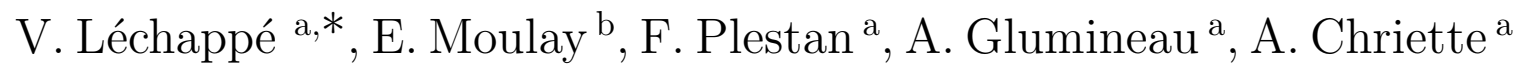 \\ ${ }^{a}$ LUNAM Université, Ecole Centrale de Nantes, IRCCyN UMR CNRS 6597, Nantes, France \\ ${ }^{\mathrm{b}}$ Xlim-SIC, Université de Poitiers - 11 Boulevard Marie et Pierre Curie - 86962 Futuroscope Chasseneuil Cedex
}

\begin{abstract}
A new predictive scheme is proposed for the control of Linear Time Invariant (LTI) systems with a constant and known delay in the input and unknown disturbances. It has been achieved to include disturbances effect in the prediction even though there are completely unknown. The Artstein reduction is then revisited thanks to the computation of this new prediction. An extensive comparison with the standard scheme is presented throughout the article. It is proved that the new scheme leads to feedback controllers that are able to reject perfectly constant disturbances. For time-varying ones, a better attenuation is achieved for a wide range of perturbations and for both linear and nonlinear controllers. A criterion is given to characterize this class of perturbations. Finally, some simulations illustrate the results.
\end{abstract}

Key words: Input delay, unknown disturbance, prediction, reduction method, Artstein reduction, predictive feedback.

\section{Introduction}

\subsection{State of the art and motivation}

A seminal work on the control of input delay systems is the well-known Smith predictor [21]. It is a frequency domain approach for SISO and open-loop stable systems. In the 80's, the Finite Spectrum Assignment (FSA) [10], [12] and the model reduction, also called Artstein reduction [1], have extended Smith's work to MIMO, openloop unstable systems. Both methods lead to the construction of a predictive feedback even though the underlying idea is different. These methods are very efficient when the delay is too large (with respect to the dynamics of the system) to be neglected and a standard (memoryless) feedback would fail. However, they suffer some drawbacks. The main problem is that an accurate model and the exact knowledge of the delay are required

\footnotetext{
* Corresponding address: IRCCyN, 1 rue de la Noë, 44321 Nantes, France Tel. +332403769 32. Fax +332403769 30 .

Email addresses:

vincent.lechappe@irccyn.ec-nantes.fr (V. Léchappé ), emmanuel.moulay@univ-poitiers.fr (E. Moulay), franck.plestan@irccyn.ec-nantes.fr (F. Plestan), alain.glumineau@irccyn.ec-nantes.fr (A. Glumineau), abdelhamid.chriette@irccyn.ec-nantes.fr (A. Chriette).
}

to have a good prediction. Consequently, these methods are not robust to model and delay uncertainties. Besides, a careless implementation of the prediction can induce the divergence of the closed loop system [15], [22]. To avoid this difficulty, the Truncated Predictor Feedback (TPF) drops the integral part [23]. It can be seen as an halfway solution between memoryless feedback and predictive feedback (memory feedback). A complete analysis of predictive control can be found in [8].

In spite of numerous works on TDS, very few articles deal with disturbance attenuation in presence of delay in the input even if there is a real interest from a practical point of view. Indeed, it is really a complex challenge even for linear systems. Some robust control methods have been extended to input delay systems. First, sliding mode control, known for its robustness in the delay-free case, has been adapted to input delay systems by using "surfaces" [17], [20] or a standard surface [3], [5]. The problem of sliding mode with relay systems is the unavoidable apparition of oscillations [4]. $H_{\infty}$ control has also been studied for input delay systems and a review is proposed in [14]. A complete analysis of this topic is also provided by [25].

To end this literature review, some references on predictive feedback (state space approach) with disturbance attenuation can be cited. Even with a known delay, it is 
not possible to reject perfectly a matched, time-varying perturbation because disturbances are unpredictable. As a result, one can only try to reduce their effects on the system. In [11], a geometric approach is used to show the existence of an output predictive feedback that minimizes the effect of the disturbance on the system. Krstic proposed a filtered predictive feedback which minimizes a cost functionnal in [7] and [9]. Recently, Polyakov et al. apply the attractive ellipsoid method to stabilize MIMO systems with time-varying delay in the input [18]. Pyrkin et al. [19] use an adaptive scheme to estimate and reject the sinusoidal disturbances on an LTI system with known delay. An adaptive control scheme allows the exact rejection of a constant disturbance in presence of constant and unknown delay in [2].

\subsection{Contribution}

In this work, disturbance information has been indirectly introduced into the prediction even though the disturbance is unknown (see Definition 8). Then, the Artstein transform is revisited with this new prediction. Thanks to this control scheme, a perfect rejection of constant perturbations can be obtained (Proposition 11). Furthermore, it is demonstrated that the new predictive scheme allows controllers to better attenuate a wide class of time-varying disturbances (see Theorem 13). To summarize, the main contributions consist in:

- designing a new prediction by indirectly including some disturbance information despite the perturbation is unknown;

- studying the modifications on the Artstein reduction method;

- defining precisely the range of validity of the new predictive scheme for time-varying disturbances.

\subsection{Paper's structure}

The paper is organized as follows. In Section 2, the problem formulation is presented and the standard predictive approach is recalled. Subsection 3.1 describes how the new prediction is obtained. Then, the reduction method is revisited in Subsection 3.2. The scope of the new scheme for time varying perturbations is worked out in Subsection 3.3. Theoretical results are confirmed by numerous simulations in Section 4. Finally, some conclusions are drawn in Section 5.

\section{Problem statement and standard predictive approach reminder}

\subsection{The standard prediction}

The considered systems are LTI systems with a delay $h$ acting on the control input $u$ and an additive perturba- tion $d$

$$
\left\{\begin{array}{l}
\dot{x}(t)=A x(t)+B u(t-h)+d(t) \\
u(t)=u_{0}(t) \quad \text { for all } t \in[-h, 0[ \\
x(0)=x_{0}
\end{array}\right.
$$

with $x(t) \in \mathbb{R}^{n}, u(t) \in \mathbb{R}^{m}, d(t) \in \mathbb{R}^{n}, A \in \mathbb{R}^{n \times n}$ and $B \in \mathbb{R}^{n \times m}$.

Assumption $1 A$ and $B$ are constant and known and the pair $(A, B)$ is controllable.

\section{Assumption $2 h>0$ is constant and known.}

Let $I \subseteq \mathbb{R}$ be an unbounded interval and $S \subseteq \mathbb{R}^{m}$ be a set. The space of locally integrable functions $\bar{u}($.) defined on $I$ and taking values into $S$ is denoted by $L_{l o c}^{1}(I, S)$.

Assumption $3 u$ is a locally integrable function: $u \in$ $L_{l o c}^{1}\left(\left[-h,+\infty\left[, \mathbb{R}^{m}\right)\right.\right.$.

Assumption $4 d$ is an unknown locally integrable function : $d \in L_{\text {loc }}^{1}\left(\mathbb{R}_{\geq 0}, \mathbb{R}^{n}\right)$.

Note that if $u$ and $d$ are bounded and measurable as in [1] then Assumptions 3 and 4 are verified.

The prediction at time $t$ of the state of the system (1) at time $t+h$, denoted $x_{p}(t)$ reads as

$$
x_{p}(t)=e^{A h} x(t)+\int_{t-h}^{t} e^{A(t-s)}[B u(s)+d(s+h)] \mathrm{d} s
$$

for all $t \geq 0$. The integral term in (2) and all the integral terms mentioned in the sequel are well defined thanks to Assumptions 3 and 4.

Expression (2) is the exact prediction of the system state. In other words, one has

$$
x_{p}(t)=x(t+h) .
$$

In order to compute the integral part of (2), the value of the perturbation on the interval $[t, t+h]$ is required. A first solution to circumvent this difficulty is to compute an approximated prediction $x_{\hat{p}}$ that does not include the effect of the disturbances

$$
x_{\hat{p}}(t)=e^{A h} x(t)+\int_{t-h}^{t} e^{A(t-s)} B u(s) \mathrm{d} s
$$

for all $t \geq 0$. The computation is feasible but $x_{\hat{p}}(t) \neq$ $x(t+h)$. As a result, there is always an error between the 
exact prediction and the approximated one. This error is given by

$$
x_{p}(t)-x_{\hat{p}}(t)=\int_{t-h}^{t} e^{A(t-s)} d(s+h) \mathrm{d} s .
$$

Remark 5 From a practical point of view, the integral term in (3) is discretized in $q$ intervals, so a buffer has to save previous $q$ values of the input from $t-h$ to $t$. The number of intervals $q$ has to be selected according to $h$ and the integration step $\tau$. Usually $q$ is chosen equal to $\frac{h}{\tau}$. The reader can refer to [15], [16], [24] for more details on practical implementation.

In next section, the Artstein reduction method is reminded in order to draw a comparison with the new predictive scheme that is going to be introduced in Subsection 3.2.

\subsection{The Artstein reduction method}

The reduction method [1] consists in transforming the system with a delayed input into a delay-free system. The definition of the so-called Artstein transformation is

$$
Z(t)=e^{-A h} x_{\hat{p}}(t) .
$$

This method has been used in the disturbance-free framework. By applying the Artstein transformation on system (1) one gets

$$
\dot{Z}(t)=A Z(t)+e^{-A h} B u(t)+d(t) .
$$

However, in the context of disturbance rejection, the alternative reduction with $x_{\hat{p}}$ is prefered. It follows that

$$
\dot{x}_{\hat{p}}(t)=A x_{\hat{p}}(t)+B u(t)+e^{A h} d(t) .
$$

In the disturbance free-case, $d(t)=0$, stabilizing $x_{\hat{p}}(t)$ is equivalent to stabilize $x(t)$ since $x_{\hat{p}}(t)=x(t+h)$. However, for disturbed systems, this is not true anymore as expressed in the following proposition.

Proposition 6 The asymptotic convergence of $x_{\hat{p}}$ to zero implies the asymptotic convergence of $x$ to $\int_{t-h}^{t} e^{A(t-s)} d(s) \mathrm{d} s$.

PROOF. If $x_{\hat{p}}$ tends to zero, it can be deduced from (4) evaluated in $t-h$ that $x_{p}(t-h)=x(t)$ tends to $\int_{t-2 h}^{t-h} e^{A(t-h-s)} d(s+h) \mathrm{d} s$. A substitution in the integral ends the proof.
If $u$ is designed on (5) such that $x_{\hat{p}}$ tends to zero then $x$ will not tend to zero even for constant perturbations. This drawback makes the approximated prediction $x_{\hat{p}}$ not compatible with input delay systems with disturbances. That is why some improvements are necessary and a new predictive scheme is developped in the next section.

\section{A new predictive scheme}

\subsection{Definition and computation}

The objective is to improve the approximated prediction $x_{\hat{p}}$ by using some disturbances information. However, these latters are unknown. The basic idea is to compare the "true" state of the system (1) at time $t, x(t)$, with its approximated prediction made at time $t-h, x_{\hat{p}}(t-h)$. Recalling that $h$ is known and constant, one has:

- if $d(t)=0$ then $x(t)-x_{\hat{p}}(t-h)=0$;

- if $d(t) \neq 0$ then $x(t)-x_{\hat{p}}(t-h) \neq 0$ and depends on $d(t)$.

In presence of perturbation, the prediction error for system (1) reads as

$$
x(t)-x_{\hat{p}}(t-h)=\int_{t-h}^{t} e^{A(t-s)} d(s) \mathrm{d} s
$$

for all $t \geq h$.

Remark $7 x_{\hat{p}}(t-h)$ is not defined for $t \in[0, h[$ but setting $x_{\hat{p}}(t-h)=x_{\hat{p}}(0)$ for all $t \in[0, h[$ implies that (6) is well defined for all $t \geq 0$ and that $x_{\hat{p}}$ is continuous at $t=0$.

The new prediction is introduced by the following definition:

Definition 8 The new prediction is defined by

$$
X_{\hat{p}}(t)=x_{\hat{p}}(t)+x(t)-x_{\hat{p}}(t-h)
$$

for all $t \geq h$, with $x_{\hat{p}}$ given by

$$
x_{\hat{p}}(t)=e^{A h} x(t)+\int_{t-h}^{t} e^{A(t-s)} B u(s) \mathrm{d} s .
$$

Note that the integral expression of (7) can be obtained combining (6) with (3). It yields to

$$
X_{\hat{p}}(t)=e^{A h} x(t)+\int_{t-h}^{t} e^{A(t-s)}[B u(s)+d(s)] \mathrm{d} s
$$


for all $t \geq h$. Comparing (8) with predictions (2) and (3), one realizes that $X_{\hat{p}}$ is an halfway prediction beetween the standard prediction and the exact one. Besides, it is clear from (7) that there is no need to know the perturbation to compute the new prediction $X_{\hat{p}}$.

Remark 9 Given that $x_{\hat{p}}(t-h)=x_{\hat{p}}(0)$ for allt $\in[0, h[$, the equality $X_{\hat{p}}(t)=x_{\hat{p}}(t)+x(t)-x_{\hat{p}}(0)$ holds for all $t \in\left[0, h\left[\right.\right.$ and guarantees that $X_{\hat{p}}$ is well defined and continuous for all $t \geq 0$.

The only difference between $(8)$ and (2) is the term $d(s)$ instead of $d(s+h)$ in the integral part. It means that (7) is almost the exact prediction in spite of the unknown disturbance. As a comparison with (4), the error between the exact prediction $x_{p}$ and the new prediction $X_{\hat{p}}$ is

$$
x_{p}(t)-X_{\hat{p}}(t)=\int_{t-h}^{t} e^{A(t-s)}[d(s+h)-d(s)] \mathrm{d} s .
$$

From errors (4) and (9), it is clear that the accurracy of the predictions depends on the magnitude of the disturbance for (4) and on the dynamics of the disturbance for (9).

As far as the implementation issues are concerned, there is no extra complexity. The only requirement is to store the values of $x_{\hat{p}}$ on the interval $[t-h, t]$ in addition to the values of $u$ on the interval $[t-h, t]$. Indeed, the new prediction is computed from the standard prediction thanks to (7). Consequently, all the results mentioned for the computation of $x_{\hat{p}}$ [15], [24] can be used to analyse the computation of $X_{\hat{p}}$. In particular, it has been shown in [24] that the approximation error can be made as small as small possible for a discretization in the $s$-domain and if the number of approximation steps is large enough.

Remark 10 For state-space systems, full state knowledge is required to be able to compute prediction (3). Similarly, the computation of the new prediction (7) requires full state knowledge. In case of partial state measurement, state reconstruction can be used as in [11] and [13]. In theses works, the prediction (3) is computed from the reconstructed state. Then, the new prediction can be computed replacing $x_{\hat{p}}$ in (7) by the "reconstructed prediction". Since this paper focuses on the comparison with the Artstein reduction (that considers full state knowledge), we will not go into details on the observer-predictor structure. Next results hold when the observation error tends to zero.

\subsection{The reduction method with the new prediction}

In this subsection, the objective is to study how (5) is modified when the transformation is the new prediction. As in Subsection 2.2, a transformation is carried out to turn the system into a delay-free one with respect to the input. The dynamics of $X_{\hat{p}}$ is

$\dot{X}_{\hat{p}}(t)=A X_{\hat{p}}(t)+B u(t)+d(t)+e^{A h}[d(t)-d(t-h)]$

for all $t \geq h$. When $d(t)=d(t+h)$, one has $X_{\hat{p}}=x_{p}$ and the last term of (10) cancels. In this case, (10) is exactly equal to (1) but without delay. An interesting property of this new prediction is stated in the next proposition.

Proposition 11 For constant disturbances, the asymptotic convergence of $X_{\hat{p}}$ to zero implies the asymptotic convergence of $x$ to zero.

PROOF. If $X_{\hat{p}}$ tends to zero, it follows from (9) that $x_{p}(t)=x(t+h)$ tends to $\int_{t-h}^{t} e^{A(t-s)}[d(s+h)-d(s)] \mathrm{d} s$. For constant perturbations, $d(s+h)=d(s)$ so $x(t+h)$ tends to zero and $x(t)$ as well.

In this case, stabilizing $X_{\hat{p}}(t)$ implies that $x(t)$ is stabilized to the same value. This is a major improvement of this new predictive scheme. Table 1 gathers the three different predictive appoaches discussed in this paper.

\subsection{Analysis of the new predictive scheme for time- varying disturbances}

In order to make a qualitative analysis of the perturbation attenuation properties, the next assumption is required

Assumption $12 d: \mathbb{R}_{\geq 0} \mapsto \mathbb{R}^{n}$ is an unknown locally integrable function such that for all $t \geq 0$,

$$
\|d(t)\| \leq d_{\max }<+\infty
$$

and for all $t \geq h$,

$$
\|d(t)-d(t-h)\| \leq h D_{\max }<+\infty .
$$

The bounds $d_{\max }$ and $D_{\max }$ are supposed to be known.

Thanks to Propositions 6 and 11, the improvement of the new predictive scheme has been clearly highlighted for constant perturbations. For time varying perturbations $\left(D_{\max }>0\right)$, asymptotic stability cannot be achieved anymore, only stability within a ball around the origin is possible. The objective of this subsection is to study the influence of the prediction scheme on the error bound. 
Table 1

Comparison of the predictive schemes

\begin{tabular}{|c|c|c|c|}
\hline & $\begin{array}{c}\text { STANDARD PREDICTIVE } \\
\text { SCHEME }\end{array}$ & $\begin{array}{c}\text { NEW PREDICTIVE } \\
\text { SCHEME }\end{array}$ & $\begin{array}{c}\text { EXACT PREDICTIVE } \\
\text { SCHEME }\end{array}$ \\
\hline Prediction & $x_{\hat{p}}(t)=e^{A h} x(t)+$ & $X_{\hat{p}}(t)=x_{\hat{p}}(t)+x(t)-x_{\hat{p}}(t-h)$ & $e^{A h} x(t)+$ \\
\hline \multirow{2}{*}{$\begin{array}{c}t \\
\text { Reduced }\end{array}$} & $\int_{t-h}^{t} e^{A(t-s)} B u(s) \mathrm{d} s$ & see eq. (8) for the integral expression $\int_{t-h}^{t} e^{A(t-s)}[B u(s)+d(s+h)] \mathrm{d} s$ \\
\end{tabular}

Let $f$ be a Lipschitz continuous function and assume the control

$$
u(t)=f\left(x_{\hat{p}}(t)\right)
$$

is such that the origin is a globally exponentially stable equilibrium point of the nominal system (5) with $d(t)=0$. The function $f: \mathbb{R}^{n} \rightarrow \mathbb{R}^{m}$ is locally Lipschitz and $x_{\hat{p}}$ is continuous so Assumption 3 holds. Besides, Theorem 4.14 in [6] guarantees the existence of a Lyapunov function $V\left(x_{\hat{p}}\right)$ that satisfies

$$
\begin{aligned}
& c_{1}\left\|x_{\hat{p}}\right\| \leq V\left(x_{\hat{p}}\right) \leq c_{2}\left\|x_{\hat{p}}\right\| \\
& \dot{V}\left(x_{\hat{p}}\right) \leq-c_{3}\left\|x_{\hat{p}}\right\| \\
& \left\|\frac{d V}{d x_{\hat{p}}}\right\| \leq c_{4}\left\|x_{\hat{p}}\right\|
\end{aligned}
$$

with $c_{1}, c_{2}, c_{3}$ and $c_{4}$ positive constants. In addition, the perturbation of system (5) is bounded and the following maximization holds

$$
\left\|e^{A h} d(t)\right\| \leq\left\|e^{A h}\right\| d_{\max }, \quad \forall t \geq 0 .
$$

Therefore, the assumptions of Lemma 9.4 in [6] are fulfilled (equations (14) and (15)) so one deduces that for all $t \geq 0$

$$
\left\|x_{\hat{p}}(t)\right\| \leq \beta\|x(0)\| e^{-\alpha t}+\gamma\left\|e^{A h}\right\| d_{\max }
$$

with $\alpha, \beta$ and $\gamma$ positive constants that depends on $c_{1}$, $c_{2}, c_{3}$ and $c_{4}$.

Since (5) and (10) have the same form when $d(t)=0$, the controller

$$
u(t)=f\left(X_{\hat{p}}(t)\right)
$$

guarantees that $X_{\hat{p}}=0$ is a globally exponentially stable equilibrium point of the nominal system (10) with $d(t)=$ 0 . Similarly to $u(t)=f\left(x_{\hat{p}}(t)\right)$, Assumption 3 is verified for $u(t)=f\left(X_{\hat{p}}(t)\right)$. Besides, inequalities (14) still holds for $X_{\hat{p}}$ :

$$
\begin{aligned}
& c_{1}\left\|X_{\hat{p}}\right\| \leq V\left(X_{\hat{p}}\right) \leq c_{2}\left\|X_{\hat{p}}\right\| \\
& \dot{V}\left(X_{\hat{p}}\right) \leq-c_{3}\left\|X_{\hat{p}}\right\| \\
& \left\|\frac{d V}{d X_{\hat{p}}}\right\| \leq c_{4}\left\|X_{\hat{p}}\right\| .
\end{aligned}
$$

From Assumption 12, the inequality

$$
\left\|d(t)+e^{A h}[d(t)-d(t-h)]\right\| \leq d_{\max }+h\left\|e^{A h}\right\| D_{\max },
$$

is verified for all $t \geq h$. Relations (18) and (19) comply with the assumptions of Lemma 9.4 in [6] so the following inequality is obtained

$$
\left\|X_{\hat{p}}(t)\right\| \leq \beta\|x(0)\| e^{-\alpha t}+\gamma\left[d_{\max }+h\left\|e^{A h}\right\| D_{\max }\right]
$$

for all $t \geq h$. The constants $\alpha, \beta$ and $\gamma$ are the same as in (16) because they only depend on the form of the undisturbed system. As it has been mentioned before, systems (5) and (10) have the same representation $\dot{\chi}=$ $A \chi+B u(t)$ when there is no perturbation (Reduced system in Table 1). Then from the evaluation of (4) and (9) in $t-h$, it follows that

$$
\|x(t)\| \leq\left\|x_{\hat{p}}(t-h)\right\|+\eta d_{\max }
$$

and

$$
\|x(t)\| \leq\left\|X_{\hat{p}}(t-h)\right\|+\eta h D_{\max }
$$

with $\eta=\left\|\int_{-h}^{0} e^{A s} \mathrm{~d} s\right\|$. As a result, if (1) is controlled by the feedback $u(t)=f\left(x_{\hat{p}}\right)$, the inequality

$$
\|x(t)\| \leq \beta\|x(0)\| e^{\alpha h} e^{-\alpha t}+\underbrace{\left[\eta+\gamma\left\|e^{A h}\right\|\right] d_{\max }}_{r_{1}}
$$


holds and, if (1) is controlled by the feedback $u(t)=$ $f\left(X_{\hat{p}}\right)$, the inequality

$\|x(t)\| \leq \beta\|x(0)\| e^{\alpha h} e^{-\alpha t}+\underbrace{\gamma d_{\max }+\left[\eta+\gamma\left\|e^{A h}\right\|\right] h D_{\max }}_{r_{2}}$

is verified. This proves the following theorem.

Theorem 13 Consider system (1), predictor-controllers (3)-(13) and (7)-(17), resulting in error bounds respectively $r_{1}$ in (22) and $r_{2}$ in (23). If the bounds on $d(t)$ comply with the relations

$$
\frac{d_{\max }}{D_{\max }}>h \frac{\left\|e^{A h}\right\|}{\left\|e^{A h}\right\|-1},
$$

then one gets $r_{2}<r_{1}$.

Note that the inequality $\left\|e^{A h}\right\|>1$ is always true since $h>0$. Theorem 13 and equations (22) and (23) show that the construction of a Lipschitz controller with the new prediction $X_{\hat{p}}$ given by (17) leads to a smaller error bound than designing a controller with the standard prediction (13). Consequently, the new predictive scheme is said to better attenuate the disturbances than the standard one.

Note that some additional assumptions on the eigenvalues of $A$ would be necessary to determine whether or not (24) is fulfilled for a given $h$. Besides, systems are usually such that $\left\|e^{A h}\right\|>>1$ so (24) can be approximated by

$$
\frac{d_{\max }}{D_{\max }}>h \text {. }
$$

In this case, the attenuation only depends on the dynamics of the perturbation with respect to the delay size. However, the converse of Theorem 13 is not true; even if the perturbation does not comply with (24), a controller using the new prediction can better attenuate the perturbation but it is not guaranteed. Theorem 13 applies to a wide range of linear and nonlinear controllers. This gives the possibility to robustly stabilize $X_{\hat{p}}$ at zero. However, even if $X_{\hat{p}}$ converges to zero, there is an inevitable error, $\eta h D_{\max }$ in (21), independent from the control and that cannot be reduced for time-varying disturbances.

\section{Simulation}

\subsection{Model presentation}

A second order perturbed system has been chosen to illustrate the results. Its state space representation is

$$
\dot{x}(t)=\left[\begin{array}{cc}
0 & 1 \\
-a_{0} & -a_{1}
\end{array}\right] x(t)+\left[\begin{array}{l}
0 \\
1
\end{array}\right] u(t-h)+\left[\begin{array}{c}
0 \\
d(t)
\end{array}\right]
$$

with $a_{0}=9$ and $a_{1}=-3$. The parameters chosen for all the simulations are $h=0.5 \mathrm{~s}$ and $x(0)=[1.5,1]^{T}$. In the sequel, the components of a vector are denoted by the subscript "i". For instance, one has $x_{\hat{p}}=\left[x_{\hat{p} 1}, x_{\hat{p} 2}\right]^{T}$.

\subsection{Comparison of the schemes for constant distur- bances}

In this subsection, Propositions 6 and 11 are illustrated. The objective consists in making the predictions $x_{\hat{p}}$ and $X_{\hat{p}}$ tend to zero and, in evaluating the behaviour of $x$. For that reason, two PID controllers are chosen to robustly stabilize delay free systems (5) and (10). Two simulations have been carried out with piecewise constant perturbation:

- Simulation 1. The controller

$$
u(t)=k_{p} x_{\hat{p} 1}(t)+k_{d} \dot{x}_{\hat{p} 1}(t)+k_{i} \int_{0}^{t} x_{\hat{p} 1}(s) d s
$$

forces $x_{\hat{p}}$ to tend to zero.

- Simulation 2. The controller

$$
u(t)=k_{p} X_{\hat{p} 1}(t)+k_{d} \dot{X}_{\hat{p} 1}(t)+k_{i} \int_{0}^{t} X_{\hat{p} 1}(s) d s
$$

forces $X_{\hat{p}}$ to tend to zero.

Results are displayed on Figures 1 and 2. For simulation 1 , the predictive state $x_{\hat{p}}$ is indeed stabilized at zero but the real state $x$ converges to a constant. The value of this constant is explicitely given in Proposition 6. For simulation 2 , both state $x$ and prediction $X_{\hat{p}}$ go to zero as it was stated in Proposition 11; the perturbation is perfectly rejected. The PID coefficients are the same in both cases, $k_{p}=45, k_{d}=18$ and $k_{i}=60$. It is shown that synthesizing robust control on system (10) (simulation 2) is much more efficient than synthesizing on system (5) (simulation 1). As a comparison, in [7], the controller obtained by filtering the standard predictive feedback is not able to reject perfectly a constant disturbance. It has been illustrated that asymptotic stabilization is obtained for piecewise constant disturbance. For time-varying perturbation, only practical stability can be achieved as it will be shown in the next subsection.

\subsection{Comparison of the schemes for time-varying dis- turbances}

The objective is to illustrate Theorem 13. A state feedback control has been chosen to stabilize the system: the function $f$ defined in the proof of $(13)$ is $f(\chi)=K \chi$ with $K \in \mathbb{R}^{m \times n}$ with $\chi$ being $x_{\hat{p}}$ or $X_{\hat{p}}$. It is reminded that any kind of robust controllers for delay-free systems can be designed on the reduced models (5) and (10). The two following controllers are going to be compared: 


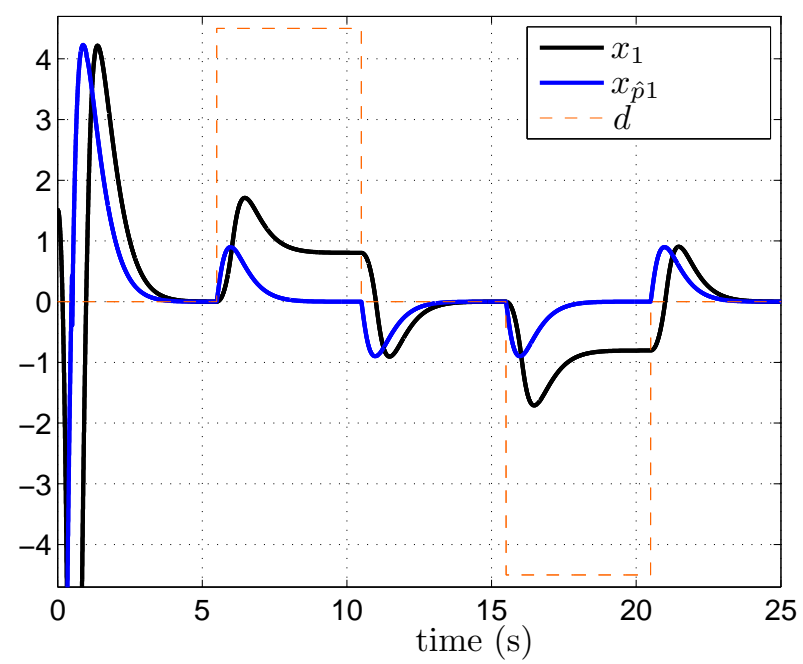

Fig. 1. Simulation 1: comparison between $x_{\hat{p} 1}$ and $x_{1}$ with piecewise constant perturbation

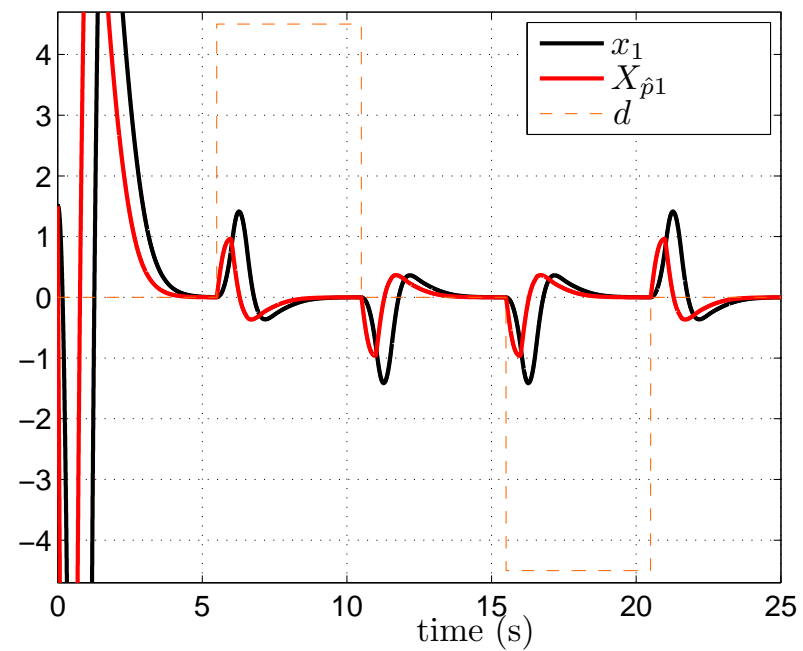

Fig. 2. Simulation 2: comparison between $X_{\hat{p} 1}$ and $x_{1}$ with piecewise constant perturbation

- the standard predictive state feedback control

$$
u(t)=-K x_{\hat{p}}(t)
$$

- the new predictive state feedback control

$$
u(t)=-K X_{\hat{p}}(t) .
$$

For a fair comparison, the gain matrices $K$ are the same for both controllers, $K=[45,18]$. In the delay-free case and without disturbances, the feedback $u(t)=-K x(t)$ drives $(26)$ to zero in about $1.5 \mathrm{~s}$. Consequently, the delay $h=0.5 \mathrm{~s}$ is very large with respect to the dynamics of the delay-free system. In this case, the system cannot be stabilized with a memoryless feedback control even in the disturbance-free case. Consequently, it is necessary to use a predictive feedback. The signal $d$ is defined as

$$
d(t)=3 \sin (0.5 t)
$$

so $d_{\max }=3$ and $D_{\max }=1.5$. For this system, $\left\|e^{A h}\right\| \approx$ 7.32 so $h \frac{\left\|e^{A h}\right\|}{\left\|e^{A h}\right\|-1} \approx 0.58$ then criterion (24) is verified. Figure 3 confirms that (28) attenuates better the perturbation than (27) because $\|x(t)\|$ with controller (27) is smaller than $\|x(t)\|$ with controller (28) during the steady state.

When perturbations are very fast time-varying, a lowpass filter can be added to the standard feedback (27), as in [7], to achieve a better attenuation of the disturbance.

These simulations confirm that designing a controller thanks to the new predictive scheme, i.e, a controller using $X_{\hat{p}}$, leads to the perfect rejection of constant perturbations and a better attenuation of time-varying perturbations than a controller using $x_{\hat{p}}$ (provided that (24) holds).
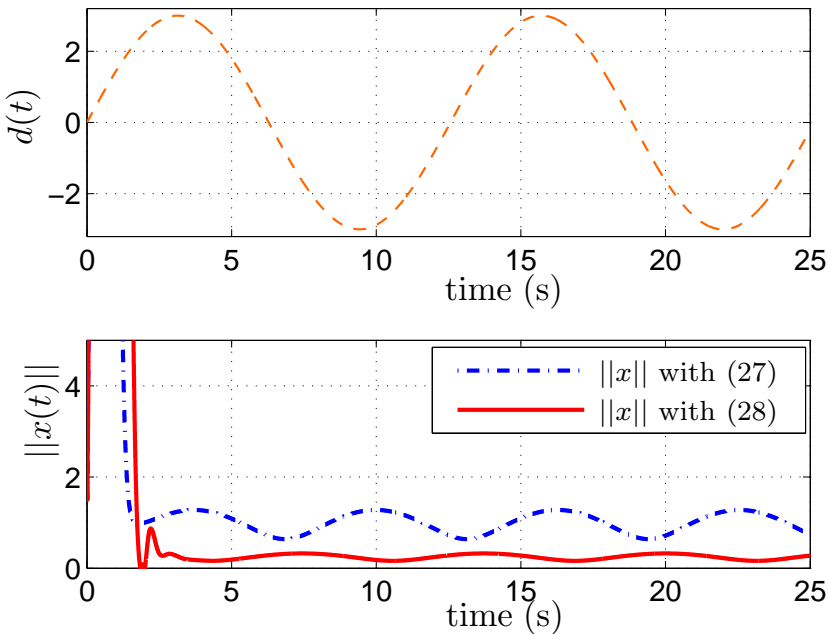

Fig. 3. Disturbance and state norm comparison

\section{Conclusion}

It has been shown that the perturbation effects can be indirectly included in a new prediction even though disturbances are unknown. Then, the modifications on the reduction method have been investigated. It has been proved that controllers designed using the new predictive approach perform a better disturbance attenuation for a wide class of disturbances. All the results are illustrated by simulations. 


\section{References}

[1] Z. Artstein. Linear systems with delayed controls: A reduction. IEEE Transactions on Automatic Control, 27(4):869-879, 1982.

[2] D. Bresch-Pietri, J. Chauvin, and N. Petit. Adaptive control scheme for uncertain time-delay systems. Automatica, 48(8):1536 - 1552, 2012.

[3] O. Camacho, R. Rojas, and W. Garcia-Gabin. Some long time delay sliding mode control approaches. ISA Transactions, 46(1):95 - 101, 2007.

[4] L Fridman, E Fridman, and E Shustin. Steady modes in relay systems with delay. In Wilfrid Perruquetti and JeanPierre Barbot, editors, Sliding mode control in engineering, Automation and Control Engineering. CRC Press, 2002.

[5] X. Han, E. Fridman, and S.K. Spurgeon. Sliding mode control in the presence of input delay: A singular perturbation approach. Automatica, 48(8):1904 - 1912, 2012.

[6] H.K. Khalil. Nonlinear Systems. Prentice Hall PTR, 2002.

[7] M. Krstic. Lyapunov tools for predictor feedbacks for delay systems: Inverse optimality and robustness to delay mismatch. Automatica, 44(11):2930 - 2935, 2008

[8] M. Krstic. Delay compensation for nonlinear, adaptive, and PDE systems. Systems and Control: Fundations and Applications. Springer, 2009.

[9] M. Krstic. Compensation of infinite-dimensional actuator and sensor dynamics. IEEE Control Systems, 30(1):22-41, 2010.

[10] W. Kwon and A. Pearson. Feedback stabilization of linear systems with delayed control. IEEE Transactions on Automatic Control, 25(2):266-269, 1980.

[11] M. Di Loreto, J.-J. Loiseau, and J.-F. Lafay. Disturbance attenuation by dynamic output feedback for input-delay systems. Automatica, 44(8):2202 - 2206, 2008.

[12] A. Manitius and A.W. Olbrot. Finite spectrum assignment problem for systems with delays. IEEE Transactions on Automatic Control, 24(4):541-552, 1979.

[13] L. Mirkin and N. Raskin. Every stabilizing deadtime controller has an observer-predictor-based structure. Automatica, 39(10):1747 - 1754, 2003.

[14] L. Mirkin and G. Tadmor. $H_{\infty}$ control of system with i/o delay: a review of some problem oriented methods. IMA Journal of Mathematical Control and Information, 19(1 and 2):185-199, 2002.

[15] S. Mondie and W. Michiels. Finite spectrum assignment of unstable time-delay systems with a safe implementation. IEEE Transactions on Automatic Control, 48:2207-2212, 2003.

[16] H. Mounier and J. Rudolph. Flatness-based control of nonlinear delay systems: A chemical reactor example. International Journal of Control, 71(5):871-890, 1998.

[17] A. Polyakov. Minimization of disturbances effects in time delay predictor-based sliding mode control systems. J. Franklin Inst., 349(4):1380-1396, 2012.

[18] A. Polyakov, A. Poznyak, and J.-P. Richard. Robust output stabilization of time-varying input delay systems using attractive ellipsoid method. In Conference on Decision and Control, Firenze, Italy, 2013.

[19] A. Pyrkin, A. Smyshlyaev, N. Bekiaris-Liberis, and M. Krstic. Rejection of sinusoidal disturbance of unknown frequency for linear system with input delay. In American Control Conference, Baltimore, USA, 2010.
[20] Y.-H. Roh and J.-H. Oh. Sliding mode control with uncertainty adaptation for uncertain input-delay systems. International Journal of Control, 73(13):1255-1260, 2000.

[21] O. J. M. Smith. Closer control of loops with dead time. Chemical Engineering Progress, 53(5):217-219, 1957.

[22] V. Van Assche, M. Dambrine, J.-F. Lafay, and J. P Richard. Some problems arising in the implementation of distributeddelay control laws. In Conference on Decision and Control, Phoenix, USA, 1999.

[23] S. Y. Yoon and Z. Lin. Truncated predictor feedback control for exponentially unstable linear systems with time-varying input delay. Systems and Control Letters, 62(10):837 - 844, 2013.

[24] Q.-C. Zhong. On distributed delay in linear control lawspart I: discrete-delay implementations. IEEE Transactions on Automatic Control, 49(11):2074-2080, 2004.

[25] Q.-C. Zhong. Robust control of time-delay systems. Springer, 2006. 